\title{
DRUG-ADMINISTERING PERSONS' EXPOSURE TO ORAL ANTICANCER DRUGS TO BE ADMINISTERED THROUGH A TUBE
}

\author{
MASAHIRO MURAKAMI ${ }^{1}$, SATOKO KATSURAGI ${ }^{1}$, MASAKO OHNO ${ }^{1}$, MAKOTO SHIGEMATSU ${ }^{2}$, AYUMI KISHI ${ }^{3}$, \\ YOSHITO ZAMAMI ${ }^{4}$, FUSAO KOMADA ${ }^{5}$, NAOMI KURATA ${ }^{6}$, MANABU AMANO ${ }^{1 *}$
}

${ }^{1}$ Section of Clinical Pharmaceutics, School of Pharmacy, Hyogo University of Health Sciences, Japan. ${ }^{2}$ Faculty of Pharmacy, Tanimukai Hospital, Japan. ${ }^{3}$ Section of Fundamental Nursing, School of Nursing, Hyogo University of Health Sciences, Japan. ${ }^{4}$ Department of Clinical Pharmacy, Tokushima University Graduate School, Japan. ${ }^{5}$ School of Pharmacy, Himeji Dokkyo University, Japan. ${ }^{6}$ School of Pharmacy, Showa University, Japan. Email: mbam@huhs.ac.jp

Received: 24 June 2016, Revised and Accepted: 16 September 2016

ABSTRACT

Objective: The objective of this study was to quantitatively evaluate anticancer drug exposure of non-health care professionals who administer drugs through a tube employing a method devised by us.

Methods: The subjects were 30 general volunteers aged 22-84 years. They wore gloves and administered Indian ink, simulating an anticancer drug, to a multipurpose adult human-type patient care simulator through a tube using 5 types of syringe, and the area stained with Indian ink was measured.

Results: When comparing the number of pixels among the syringes regardless of age, Syringe B showed the lowest number $\left(11.8 \pm 3.1 \mathrm{~cm}^{2}\right)$, and there was a significant difference between Syringes B and E. Furthermore, we compared the total number of pixels in each age group regardless of the type of syringe. In the 20-year-old group, it was the lowest $\left(10.9 \pm 2.3 \mathrm{~cm}^{2}\right)$ showing significant differences in comparison with the other groups. When Syringe B was used, the number of pixels was markedly lower than on adopting the other syringes.

Conclusion: It was clarified that the level of exposure to anticancer drugs markedly varies depending on the type of syringe and age. It was also clarified that the method to evaluate exposure to anticancer drugs using Indian ink devised by us is simple and useful.

Keywords: Oral anticancer drugs, Simple suspension method, Drug-administering persons' exposure.

(C) 2016 The Authors. Published by Innovare Academic Sciences Pvt Ltd. This is an open access article under the CC BY license (http://creativecommons. org/licenses/by/4. 0/) DOI: http://dx.doi.org/10.22159/ajpcr.2016.v9s3.13040

\section{INTRODUCTION}

Recently, many oral anticancer drugs have been developed, facilitating cancer treatment at home [1]. When performing such treatment for patients who are unable to take tablets or capsules due to dysphagia, nonhealth-care professionals, such as family members, tend to administer the anticancer drugs using a tube for tubal feeding. Therefore, the simple suspension method [2] may be useful for safely administering cytotoxic medicines, such as anticancer drugs, considering the risk of drug-administering persons' exposure. However, non-health care professionals' knowledge regarding the handling of anticancer drugs or administration procedures is unclear; health damage related to anticancer-drug exposure resulting from a lack of knowledge or skills must be avoided. In Japan, characterized by the rapid aging of society, persons aged over 60 years account for approximately $70 \%$ of family caregivers [3], raising a social issue: Nursing for elderly persons by elderly caregivers. Therefore, the tubal administration of anticancer drugs by elderly persons, whose motor function is reduced in comparison with young person [4], without sufficient knowledge or skills may further increase the risk of exposure.

For evaluation of the anticancer drug exposure of persons who administer drugs, a method using fluorescein sodium has been reported [5]. Anticancer drug exposure of health-care professionals is evaluated using this method and measures to reduce exposure are taken based on the results. On the other hand, anticancer drug exposure of non-health care professionals who administer drugs has not been investigated in detail. The detection of very low exposure levels and micro spots using the fluorescein sodium method is difficult because it requires measurement of the number of plots emitting fluorescence induced by ultraviolet light irradiation [6] Moreover, to quantitatively evaluate scattered fluorescein sodium, it is necessary to collect it by washing with purified water and measure it using a fluorescence spectrophotometer. Thus, we devised a method to quantitatively evaluate the anticancer drug exposure level using Indian ink, which is safe and can be easily obtained, without the use of ultraviolet light and expensive devices such as a fluorescence spectrophotometer. We investigated the influence of the age and syringe used on anticancer drug exposure of non-health care professionals who administered drugs through a tube, which has not previously been reported.

\section{METHODS}

The subjects

The subjects were 10 volunteers aged 22-24 years (20-year-old group), 10 aged 45-54 years (50-year-old group), and 10 aged 75-84 years (80-year-old group).

\section{The study contents}

Initially, the grip strength was measured to collect basic data, and then, this study was conducted according to the following procedures: The study outline is shown in Fig. 1.

Initially, the subjects wore gloves and administered $20 \mathrm{~mL}$ of Indian ink to an adult training model (Sakura II, Kyoto Kagaku Co., Ltd). For administration, 5 kinds of syringe (Syringes A to E) produced by different manufacturers (Table 1 ) were used, and administration was performed twice using the respective syringes.

In the phase of preparation, we (investigators) placed Indian ink in each syringe and handed it to the subjects before administration. After the completion of administration, the gloves were removed and any attached Indian ink was sufficiently dried. 
The count of pixel numbers

Subsequently, the gloves were cut to open them flat using scissors, and images of them were input into a personal computer using a scanner. After monochromatic processing with Gimp 2.8 (The GIMP Team), pixels at the sites of ink attachment were counted using a pixel counter (Hals Factory), and used as a parameter of exposure to anticancer drugs. When the resolution is $300 \mathrm{ppi}$, the number of pixels per $1 \mathrm{~cm}$ is 118 , and thus, the number per $1 \mathrm{~cm}^{2}$ is 13,924 . Referring to this number, the area was calculated from the number of pixels and used as an index of anticancer drug exposure.

\section{The ethical consideration}

Before this study, its contents were sufficiently explained to the subjects using an explanatory document, and written informed consent was obtained. The protocol of this study was approved by the Ethics Review Board of Hyogo University of Health Sciences (Approval No. 14034).

\section{Statistical analysis}

For statistical analysis, IBM SPSS version 22.0 software was used, and the Games-Howell method was adopted. A p-value of 0.05 was regarded as significant. Date was represented as mean \pm standard deviation.

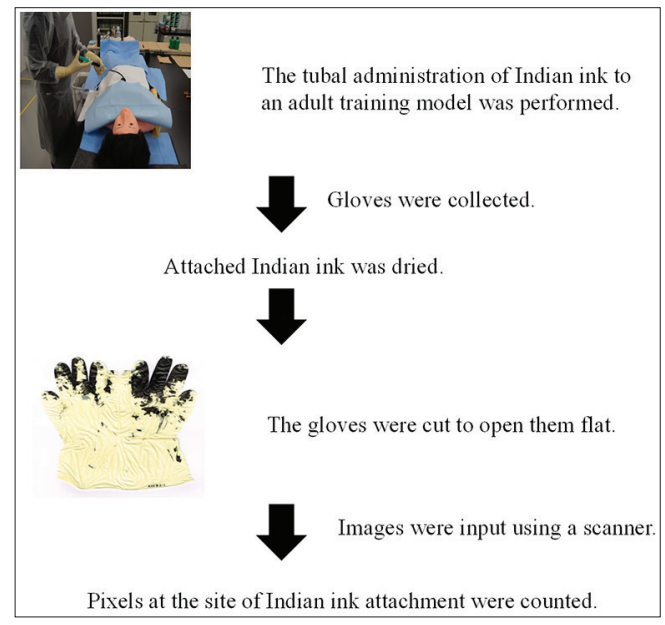

Fig. 1: Study outline

\section{RESULTS}

\section{The data for grip strength}

The mean ages of the subjects in the 20-, 50-, and 80-year-old groups were $22.7 \pm 0.8,49.9 \pm 2.5$, and $78.4 \pm 3.2$ years, respectively. In the 20 -year-old group, the mean grip strength of the left and right hands was $29.2 \pm 9.1$ and $31.3 \pm 10.9 \mathrm{~kg}$, respectively. In the 50 -year-old group, the values were $26.0 \pm 10.3$ and $27.8 \pm 9.5 \mathrm{~kg}$, respectively. In the 80 -yearold group, they were $24.1 \pm 8.1$ and $26.0 \pm 7.5 \mathrm{~kg}$, respectively.

\section{The number of pixels among the syringes}

We compared the total number of pixels on the first and second sessions of administration among the syringes regardless of age. The number of pixels for Syringe B was the lowest $\left(11.8 \pm 3.1 \mathrm{~cm}^{2}\right)$ showing a significant difference in comparison with Syringe $E$ ( $p=0.006$, Fig. $2 d$ ). With respect to age, the number of pixels for Syringe B was the lowest $\left(0.8 \pm 0.3 \mathrm{~cm}^{2}\right)$ in the 20-year-old group, showing a significant difference in comparison with Syringe E ( $\mathrm{p}=0.01$, Fig. 2a). In the 50-year-old group, the number of pixels for Syringe B was the lowest $\left(7.1 \pm 2.4 \mathrm{~cm}^{2}\right)$, showing a significant difference in comparison with Syringe $E(p=0.001$, Fig. 2b). In the 80 -year-old group, there were no significant differences among the syringes (Fig. 2c).

The number of pixels with respect to the frequency of administration

With respect to each syringe, we compared the number of pixels on the first session of administration with that on the second session. Overall, the number of pixels for Syringe B on the first session was $14.4 \pm 4.7 \mathrm{~cm}^{2}$ and that on the second session was $9.1 \pm 4.0 \mathrm{~cm}^{2}$, showing a significant decrease ( $p=0.043$, Fig. $3 d$ ). Furthermore, the number of pixels for Syringe $C$ on the first and second sessions was $27.6 \pm 7.0 \mathrm{~cm}^{2}$ and $18.2 \pm 8.7 \mathrm{~cm}^{2}$, respectively, showing a significant decrease ( $p=0.016$, Fig. $3 d$ ). With respect to age, the number of pixels for Syringe $\mathrm{E}$ on the first and second sessions in the 20-year-old group was $43.6 \pm 16.4 \mathrm{~cm}^{2}$ and $9.6 \pm 4.0 \mathrm{~cm}^{2}$, respectively, showing a significant decrease ( $p=0.014$, Fig. $3 a$ ). In the other groups, no syringe showed any significant differences between the first and second sessions (Fig. $3 \mathrm{~b}$ and 3c).

The number of pixels for all syringes

We compared the total number of pixels for all syringes on the first and second sessions among the groups. In the 20-year-old group, it was the

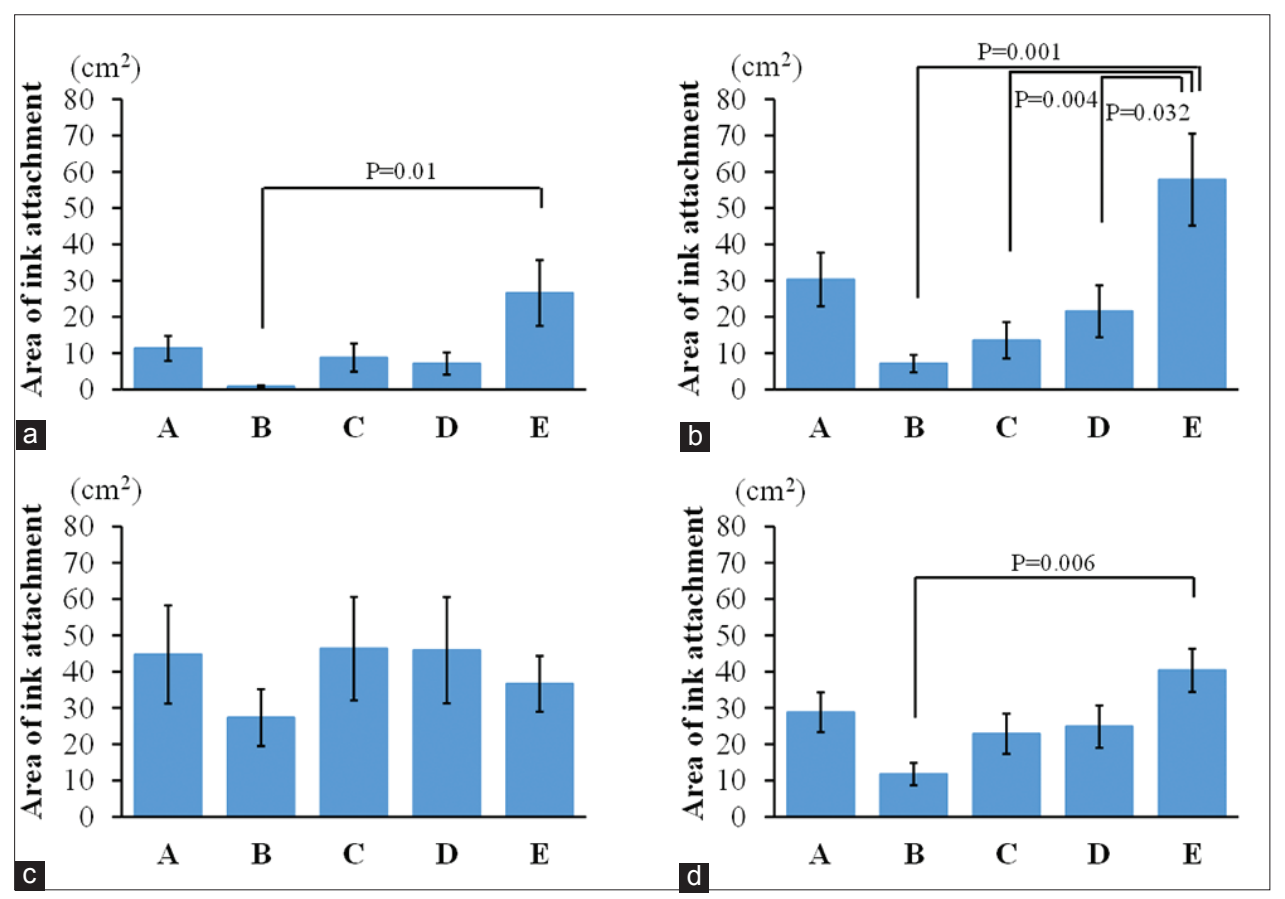

Fig. 2: Comparison of the number of pixels among the syringes, (a) 20-year-old group ( $\mathrm{n}=10)$, (b) 50-year-old group (n=10), (c) 80 -yearold group ( $n=10)$, (d) Overall $(n=30)$, Date show the mean \pm standard deviation 


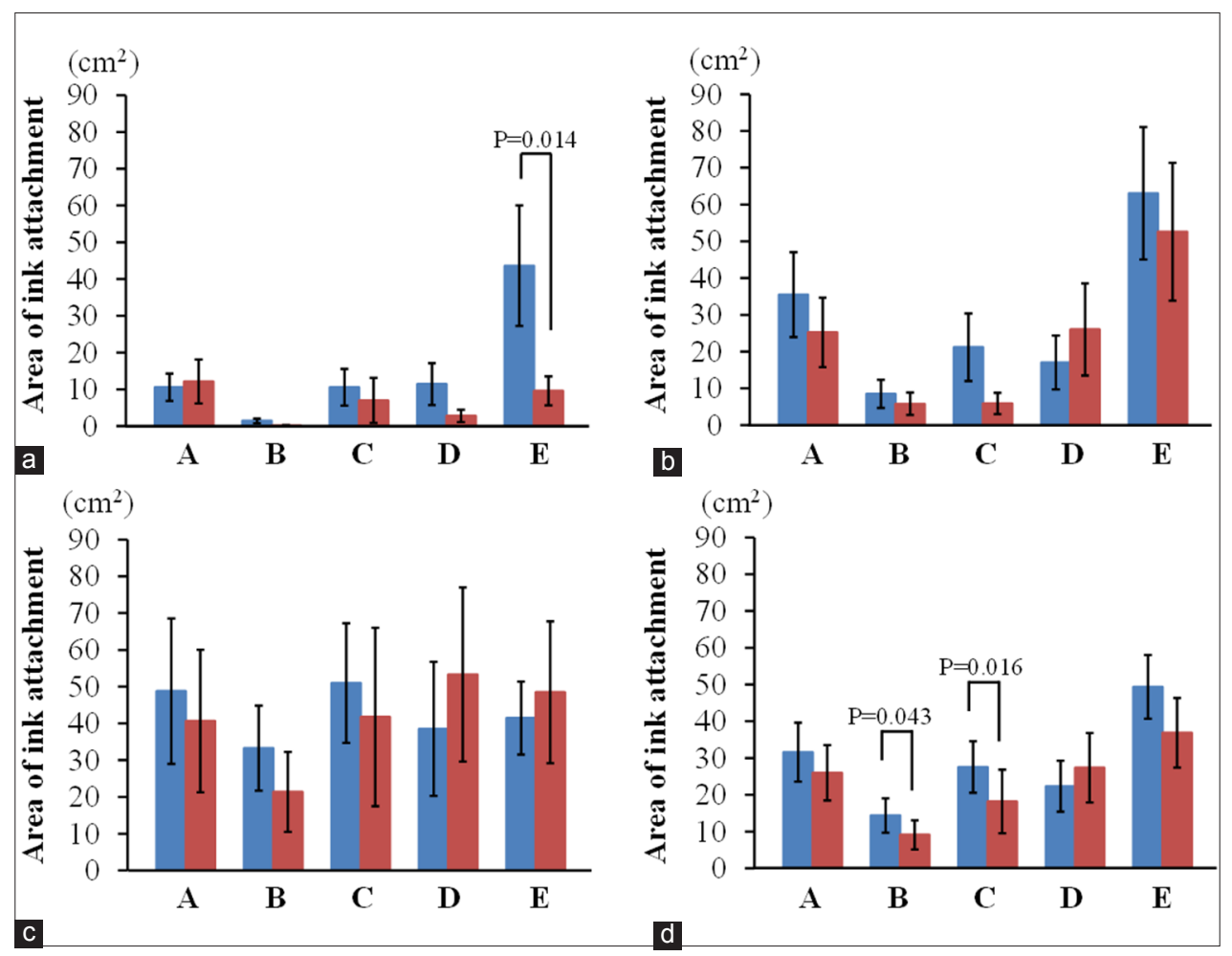

Fig. 3: Number of pixels with respect to the frequency of administration, (a) 20-year-old group (n=10), (b) 50-year-old group (n=10), (c) 80-year-old group $(n=10)$, (d) Overall $(n=30)$, $n$ : First session of administration, $n$ : Second session of administration, Date show the mean \pm standard deviation

lowest $\left(10.9 \pm 2.3 \mathrm{~cm}^{2}\right)$ showing significant differences in comparison with the other groups ( $\mathrm{p}=0.002, \mathrm{p}<0.001$, Fig. 4 ).

In each group, the total number of pixels for all syringes was compared between the first and second sessions of administration. In the 20-yearold group, the number of pixels on the first session was $15.5 \pm 4.1 \mathrm{~cm}^{2}$, and that on the second session was $6.3 \pm 1.9 \mathrm{~cm}^{2}$, showing a significant decrease ( $\mathrm{p}=0.037$, Fig. 5). In the 50-year-old group, it was $29.1 \pm 5.5$ and $23.1 \pm 5.4 \mathrm{~cm}^{2}$, respectively, showing a significant decrease $(\mathrm{p}=0.002$, Fig. 5).

\section{DISCUSSION}

Other than the method using fluorescein sodium, a method using aqueous dye-based red ink has been reported but this also requires washing of the red ink-stained region and measuring the absorbance of the washing solution. In contrast, our method analyzes the Indian ink-stained area using free software being simpler than previously reported methods. In addition, exposure can be visually confirmed without using any specific device, showing that the method is very useful. Furthermore, visually presenting the risk of exposure to patients' families, who are non-health care professionals, may be very effective.

When comparing the number of pixels among the syringes, that for Syringe B was markedly lower than for the other syringes. As shown in Table 1 and Fig. 6, we compared the characteristics of the respective syringes. Concerning Syringe B, the inner diameter of the syringe nozzle (a) was wider than that of its neck (b), differing from the other syringes; pressure in the nozzle on pushing the syringe pump may be minimized.

This may have prevented liquid leakage at the connecting part between the syringe nozzle and tube, resulting in a small volume of Indian ink attachment. Concerning Syringe E, the syringe nozzle was present on the unilateral side, and not at the center. In addition, its length was $2.0 \mathrm{~cm}$ shorter than those of the other syringes; the subjects may have

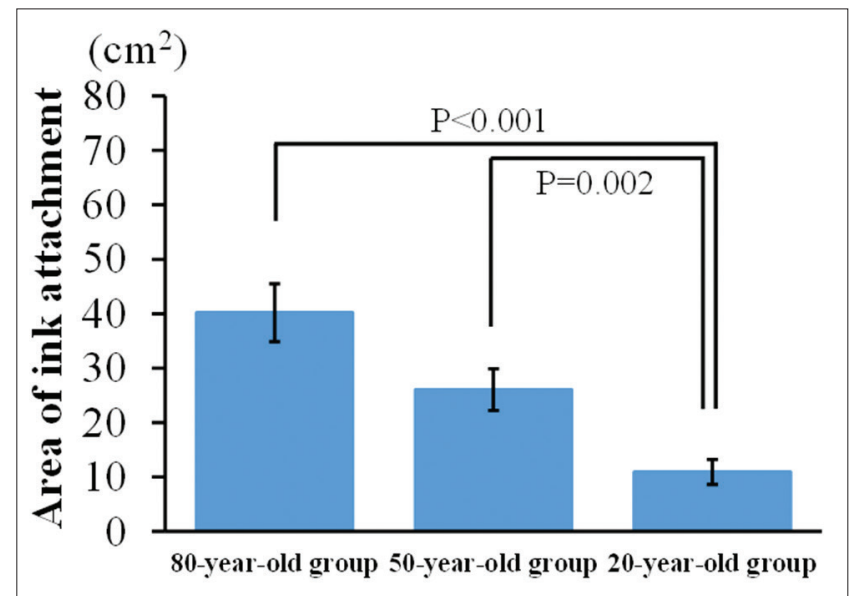

Fig. 4: Comparison of the number of pixels for all syringes among the groups $(n=30)$, Date show the mean \pm standard deviation

been frequently exposed to liquid on connecting the syringe nozzle with a tube, leading to a large volume of Indian ink attachment.

With respect to the influence of the frequency of administration, the number of pixels on the second session was lower than on the first session regardless of the age, suggesting that skills improve with the frequency of administration. However, in the 80-year-old group, the difference was less marked than in the other groups, and there was no significant difference. This was possibly associated with an agingrelated reduction of memory and understanding [7]. Therefore, it may be necessary to review administration procedures or educational tools with respect to the age.

In the 80-year-old group, the number of pixels was high regardless of the type of syringe, and there was a variation, suggesting that 
Table 1: Syringe characteristics

\begin{tabular}{lllll}
\hline Syringe & $\begin{array}{l}\text { Inner diameter of the } \\
\text { syringe nozzle up } \mathbf{( m m})\end{array}$ & $\begin{array}{l}\text { Inner diameter of the syringe } \\
\text { nozzle connecting part } \mathbf{( m m})\end{array}$ & $\begin{array}{l}\text { Length of the syringe } \\
\text { nozzle }(\mathbf{m m})\end{array}$ & Position of the syringe nozzle \\
\hline A & 4.4 & 8.0 & 30.7 & Center \\
B & 3.7 & 3.1 & 28.0 & Center \\
C & 2.6 & 4.1 & 31.4 & Center \\
D & 2.6 & 6.7 & 31.9 & Center \\
E & 1.8 & 4.0 & 10.3 & Side \\
\hline
\end{tabular}

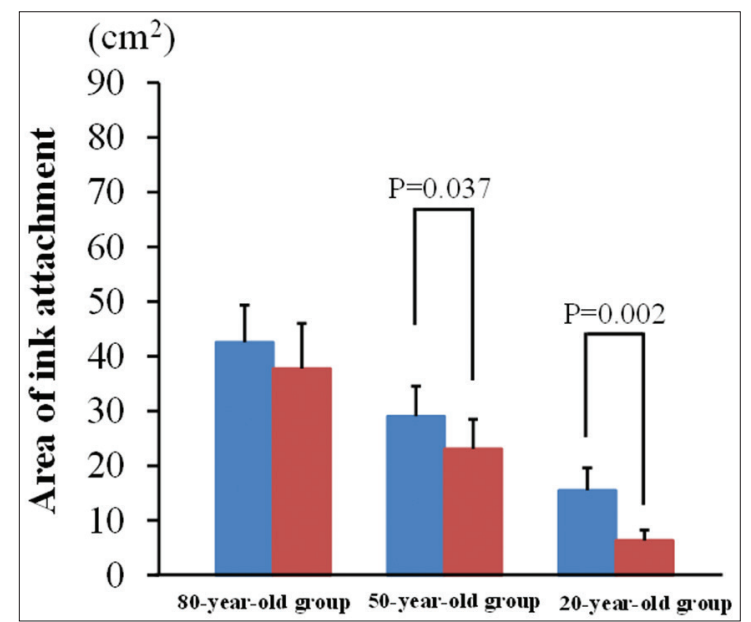

Fig. 5: Number of pixels for all syringes with respect to the frequency of administration $(n=30)$, $n$ : First session of administration, $n:$ Second session of administration, Date show the mean \pm standard deviation

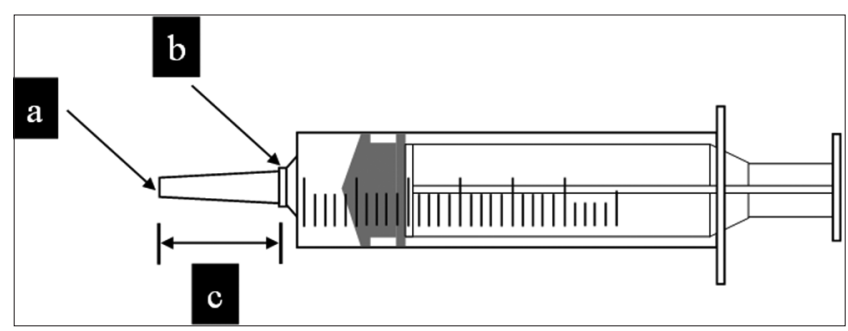

Fig. 6: The each part name of syringe nozzle, (a) The syringe nozzle tip, (b) The syringe nozzle connecting part, (c) The syringe nozzle

administration skills reduce with aging, meaning that caregivers are exposed to anticancer drugs. Kinugasa et al. reported that 80-year-old persons showed a $\leq 30 \%$ reduction of the exercise capacity involved in the motor skills of the fingers, regarding the exercise capacity at 20 years of age as 100 and that they showed a 40 to $60 \%$ reduction of the exercise capacity such as grip strength [8]. Therefore, an aging-related reduction in the motor skills of the fingers may have influenced syringe operations. On the other hand, there was no correlation between the subjects' grip strength and number of pixels; therefore, drug-administering persons' grip strength may not influence the degree of exposure.

Several studies have reported that, when administering drugs through a tube using the simple suspension method, the dose depends on the type of syringe or administration procedures $[9,10]$. This study suggests that these factors influence anticancer-drug exposure. However, the subjects were health-care professionals, and syringes of the same manufacturer with different shapes were used for comparison in these studies. Therefore, simple comparison with our study results is difficult but it was suggested that the syringe type and administration techniques influence the anticancer drug exposure level in non-health care professionals, similarly to those in health-care professionals.
Furthermore, the number of pixels did not reach zero in any subject based on the results of this study. This suggests that all subjects may be exposed to anticancer drugs when administering them through a tube regardless of the type of syringe or age.

\section{CONCLUSION}

When introducing the simple suspension method for home care, health-care professionals guide patients' families responsible for drug administration so that they can acquire the necessary skills. Since there has been no report on the exposure of non-health care professionals to anticancer drugs administered through a tube, the findings of this study cannot be compared with previous data. However, it was clarified that the anticancer drug exposure level markedly varies depending on the syringe type and age. These findings may be very useful basic data to investigate methods to select a syringe appropriate for persons who administer drugs and educate them regarding administration techniques in consideration of their age. It was also clarified that the anticancer drug exposure evaluation method using Indian ink devised by us is capable of quantitatively evaluating exposure simply and visually, being very useful.

\section{ACKNOWLEDGMENT}

This work was supported by JSPS KAKENHI Grant Number 26670260.

\section{REFERENCES}

1. Goto E, Taira S, Hosomi M, Maki C, Nishihara M, Katsumata T. The significance of cooperation between community pharmacists and hospital pharmacists using a medication notebook: Questionnaire about sharing cancer chemotherapy information between pharmacists in Takatsuki city. J Jpn Soc Hosp Pharm 2013;49:641-7.

2. Kurata N, Komastu C, Heito A, Mori Y. Examination and list of solid preparations being able to administer through feeding tubes. Jpn J Pharm Health Care Sci 2001;27:461-72.

3. Satistics and Information Department, Minister's Secretariat. Comprehensive Survey of Living Conditions 2013. Tokyo: Ministry of Health, Labour and Welfare; 2013. p. 31-4.

4. Ryushi T. Age differences of physical fitness in younger and elderly persons. J Clin Rehabil 2000;9:323-8.

5. Katsuno S, Takami S, Tatematsu M, Kaneda N. Establishment of quantitative evaluation method for anti-cancer drug handling procedures and applications using fluorescein sodium salt. Jpn J Med Pharm Sci 2014;71:417-25.

6. Higashionna T, Nawa H, Kawasaki Y, Matsushima Y, Nishimoto H, Yao $\mathrm{K}$, et al. The evaluation of countermeasures to reduced occupational exposure of anticancer drugs to nurses. J Jpn Soc Hosp Pharm 2015;51:45-8.

7. Kimbara Y, Araki A, Tamura Y, Mori S, Ito H. Treatment of old old patients with diabetes mellitus. Clin All-Round 2008;57:2483-9.

8. Kinugasa T, Nagasaki H, Ito H, Hashizume K, Furuna T, Maruyama H. Effect of aging on motor ability in men aged 18 to 83 years. J Phys Fitness Sports Med 1994;43:343-51.

9. Nagai N, Ogata F, Hayashi T, Nishiura S, Matsuoka H, Kawasaki N, et al. Preventive effect of dextrin on residue in syringe and tube obstruction for Kalimate ${ }^{\circledR}$ in the simple suspension method by feeding tube. Jpn J Pharm Health Care Sci;39:33-8.

10. Ohtani M, Yamamoto Y, Sakamaki T, Onuma A, Akagi K, Matsuda K, et al. Influence of devices and technique of administering a simple suspension on the dosage of STROMECTOL ${ }^{\circledR}$ tablets. Jpn J Pharm Health Care Sci 2012;38:78-86. 